ISSN: $1130-3743$

\title{
PRACTICUM SIN FRONTERAS: ESTUDIO DE UN CASO DE ACCIÓN Y REFLEXIÓN INTERCULTURAL Y PEDAGÓGICA
}

\author{
Practicum without borders: a case study of intercultural \\ and pedagogical action and reflection
}

\author{
Practicum sans frontières: étude d'un cas d'action \\ et de réflexion interculturelle et pédagogique
}

Ana IGLESIAS RODRÍGUEZ* y Fernando BELTRÁN LLAVADOR***

* Escuela Universitaria de Educación y Turismo de Ávila. Departamento de Didáctica, Organización y Métodos de Investigación. C/ Madrigal de las Altas Torres, 3.05003 Ávila.Correo-e: anaiglesias@usal.es

* Escuela Universitaria de Educación y Turismo de Ávila. Departamento de Filología Inglesa. C/ Madrigal de las Altas Torres, 3.05003 Ávila.Correo-e: fdob@ usal.es

Fecha de recepción: enero de 2012

Fecha de aceptación definitiva: abril de 2012

Biblid [(1130-3743) 24, 1-2012, 105-131]

\section{RESUMEN}

La formación docente del presente siglo XXI reclama una nueva contextualización de las prácticas educativas a la luz de amplios marcos globales bajo la cual hay que interpretar las especificidades culturales de los itinerarios formativos. Este estudio de caso describe algunas de las oportunidades y desafíos del Practicum Internacional de la Universidad de Salamanca para los aprendices de maestro en colaboración con instituciones educativas del Reino Unido. Más allá de la confrontación viva con la cultura como diversidad, los numerosos agentes implicados en el mismo establecieron un diálogo entre perspectivas pedagógicas diferentes que potenciaron procesos de acción docente colaborativa, interpelación mutua y reflexión conjunta 
PRACTICUM SIN FRONTERAS: ESTUDIO DE UN CASO DE ACCIÓN Y REFLEXIÓN INTERCULTURAL Y PEDAGÓGICA

de enorme valor en los ámbitos lingüístico, pedagógico y cultural. Mantenemos que ese diálogo fértil atraviesa fronteras y ya define, en sí mismo, una experiencia educativa de vinculación efectiva por cuanto sitúa la dimensión intercultural en el centro mismo de la trayectoria formativa del profesorado europeo.

Palabras clave: Practicum, formación docente, dimensión intercultural, reflexión educativa.

\section{SUMMARY}

Teacher training schemes of the present $21^{\text {st }}$ century ask for a new contextualization of good educational practice under frameworks of planetary scale which serve as tools aimed at interpreting culture-bound formation tracks designed for prospective teachers. The present case shows some of the opportunities and challenges of an International Practicum jointly organized by the University of Salamanca and several U.K. educational institutions. Besides their direct confrontation with culture as a living experience of diversity, the numerous agents involved in this programme were engaged in a dialogue between different pedagogical perspectives which crossfertilized processes of teaching collaboration, mutual inquiry and a thorough rethink of areas of common linguistic, pedagogical and cultural interest. We hold that this fruitful conversation crosses borders while it offers, per se, an educational model of cohesiveness for it places the intercultural dimension at the heart of life-long education that can shape the profile of today's European teachers.

Key words: Teaching Practicum, teaching formation, intercultural dimension, educational thinking.

\section{SOMMAIRE}

La formation des enseignants du Xxi ${ }^{m e}$ siècle réclame un nouveau contexte des pratiques éducatives à la lumière d'amples cadres globaux dans lequel il faut interpréter les spécificités culturelles des itinéraires informatifs. Ce cas présente certaines opportunités et défis du Practicum International de l'Université de Salamanque pour les apprentis d'enseignant en collaboration avec des institutions éducatives du Royaume-Uni. Au-delà de la confrontation vivante avec la culture en tant que diversité, les nombreux agents impliqués, en eux mêmes, ont établi un dialogue parmi les différentes perspectives pédagogiques qui ont renforcées des processus d'action enseignante collaborative, d'une mutuelle interpellation et d'une réflexion dans l'ensemble d'une énorme valeur dans les domaines linguistiques, pédagogiques et culturels. Nous soutenons que ce dialogue fertile traverse des frontières et définit déjà, en lui même, une liaison éducative que, dans son caractère exemplaire elle situe la dimension interculturelle dans le centre même de la trajectoire formative du professorat européen.

Mots clés: Practicum, formation enseignante, dimension interculturelle, réflexion éducative. 


\section{INTRODUCCIÓN}

La formación docente del presente siglo XXI está siendo redefinida a la luz de documentos axiales de elaboración conjunta para seguir cursos de acción de alcance global a través de Foros como el Mundial sobre la Educación reunido en Dakar, Senegal, en abril de 2000; Declaraciones como la de Bolonia, que sienta las bases para la construcción de un Espacio Europeo de Educación Superior concebido como polo de atracción para estudiantes y profesores de distintas partes del mundo; Objetivos universales para el presente milenio que persiguen, entre otras metas nobles, alcanzar la enseñanza primaria universal (González, 2011); o amplios Marcos como el Común Europeo de Referencia para el aprendizaje, enseñanza y evaluación de las lenguas. Así, el texto aprobado por el Foro Mundial sobre la Educación, en Dakar, Senegal, anteriormente aludido, con título «Educación para Todos: cumplir nuestros compromisos comunes", sostiene:

Todos los niños, jóvenes y adultos, en su condición de seres humanos tienen derecho a beneficiarse de una educación que satisfaga sus necesidades básicas de aprendizaje en la acepción más noble y más plena del término, una educación que comprenda aprender a asimilar conocimientos, a hacer, a vivir con los demás y a ser. Una educación orientada a explotar los talentos y capacidades de cada persona y desarrollar la personalidad del educando, con objeto de que mejore su vida y transforme la sociedad (UNESCO, 2000, 8).

Esas grandes visiones, por tanto, dibujan horizontes, marcan "agendas", esto es, tareas, y suponen el urgente desafío de encontrar para ellas traducción efectiva en múltiples iniciativas cuyo denominador común es el encuentro real de personas, instituciones y culturas diversas para conocerse y colaborar en proyectos educativos relevantes y de interés compartido.

Una primera, bien que determinante, transición entre el obligado conocimiento de los "nudos gordianos" de nuestro mundo, en la expresión de Federico Mayor Zaragoza (1999), y la inmersión efectiva, de orden preprofesional, en esferas locales, a modo de microcosmos educativos en el ámbito de formación docente, queda establecida por las prácticas escolares. Si bien el Practicum es uno de los capítulos de mayor potencial en la formación de los maestros, sigue considerándose uno de sus terrenos más resbaladizos, y está plagado de interrogantes que acompañan a la multitud de elementos que confluyen en el mismo: agentes, modelos, funciones, aportaciones multidisciplinares, fuentes epistemológicas, etc. (Zabalza, 2011). En efecto, por encima de cualquier otro aspecto, las prácticas profesionales de los futuros maestros se encuentran en la encrucijada de los dominios académico y profesional. Salvan la brecha, pues, entre la teoría y la práctica, entre la acción y la reflexión y suponen, en rigor, la colaboración de los ámbitos escolares y los universitarios además de confrontar el desideratum de los educadores con la realidad de las aulas, situando al alumno/maestro en una plataforma dinámica desde la que repensar su pasado escolar y proyectar su futuro docente a la luz de los retos inmediatos. Así las cosas, suscribimos la premisa de que resulta prioritario ayudar a los 
maestros en formación a formarse juicios fundamentados y a articular decisiones ética y moralmente ponderadas respecto a su práctica por medio de situaciones dialógicas y colaborativas en las que se ven obligados a afrontar dilemas y a resolver problemas que emergen en contextos vivos - "aquí y ahora"-, donde pueden adquirir competencias que, sin desdeñarlo, trascienden el mero pensar técnico sobre "qué hacer" o "qué estrategias adoptar" en el orden académico, para tomar en consideración, además, códigos culturales, fórmulas de relación, componentes emocionales, modalidades participativas o variedades discursivas con las que modular la forma y el contenido de su toma de decisiones (Orland-Barak, 2011).

Los actuales procesos de convergencia europea en el espacio educativo ponen de manifiesto similitudes y diferencias entre los diversos modelos de formación del profesorado así como en sus programas de prácticas. Ese ejercicio comparativo está sirviendo para arrojar luz sobre la necesidad imperiosa de proporcionar a los futuros docentes oportunidades para conocer, como parte de su itinerario formativo, los sistemas educativos y las realidades escolares de otros países facilitando su integración en las aulas, donde habrán de ejercitar y ampliar sus destrezas didácticas, lingüísticas e interculturales, además de asumir nuevas responsabilidades, puesto que "los recientes cambios sociales, económicos y culturales también han tenido un impacto sobre las actividades del profesorado" (EURYDICE, 2009, 14).

El presente estudio de caso (Thomas, 2011) quiere ilustrar cómo lo anterior ha venido cristalizándose en la forma de un Practicum Internacional de la Universidad de Salamanca (en adelante USAL) en colaboración con instituciones universitarias del Reino Unido -inicialmente Nottingham Trent University (en adelante NTU) y posteriormente también East Anglia University-, colegios de enseñanza primaria e instituciones educativas de ambos países, y alrededor del cual se han generado fórmulas muy diversas de formación inicial y continua: placements para estudiantes de maestro británicos y españoles en colegios de Ávila y Zamora, en España, y de Nottingham, Coventry y Norwich, en el Reino Unido; intercambios de sacos de historias en inglés y en español elaborados en colegios de los dos países; cursos de formación de lengua, cultura y educación para maestros ingleses financiados por el British Council en las Escuelas Universitarias de Educación de la USAL; creación de unidades didácticas y recursos de enseñanza entre colegios británicos y españoles (Gutiérrez, Beltrán y Durán, 2010); publicación de una guía bilingüe de prácticas escolares de elaboración conjunta (Durán, Gutiérrez y Beltrán, 2006), e intercambio de estudiantes Erasmus en el Departamento de Lenguas Modernas, Facultad de Educación de NTU y Escuelas Universitarias de Educación de la USAL, etc.

\section{El PRACTICUM INTERNACIONAL}

Los primeros proyectos, bajo el título genérico de Spanish Language and Culture Training, hicieron acreedoras de un European Award for Languages el 26 de septiembre de 2001 (Año Europeo de las Lenguas) a la Nottingham City LEA (Local Education Authority), NTU y a la USAL, siendo otorgado por Catherine 
Ashton, entonces Minister for Early Years and School Standards (Department of Education and Skills, UK) y Viviane Reading, Member of the European Comission, with responsibility for education and culture. El breve descriptor del galardón recoge sucintamente su contenido:

This partnerhsip Project between Nottingham City LEA, Nottingham Trent University and the University of Salamanca comprises three elements designed to raise awareness of Spanish language and culture: Spanish language training, student placement and school linking.

Por lo que respecta a la Guía internacional de prácticas docentes en centros de enseñanza primaria, de edición bilingüe, ésta introduce, a modo de mapa conceptual, los modelos formativos de España y del Reino Unido acudiendo a documentación de fuentes ministeriales salpicada de ejemplos clarificadores a partir de un "trabajo de campo" pedagógico. A continuación presenta el esquema organizativo del desarrollo de las prácticas docentes internacionales con descripciones de los objetivos y expectativas de los estudiantes, de sus tutores en los centros escolares y de los supervisores universitarios e incluye un detallado plan semanal de trabajo para todos ellos y pautas para la elaboración de los informes -Teaching Practice Reports o files- que han de realizar los alumnos así como plantillas de evaluación cualitativa y ejemplos completados de las mismas para los discentes españoles y británicos. Finalmente, ofrece un apéndice con sendos cuestionarios para estudiantes y profesores concebido como una herramienta para reflexionar acerca de la maraña compleja de presupuestos culturales y puntos de vista que constituyen las experiencias de enseñanza y aprendizaje, compartidas y diversas, previas y posteriores a las prácticas escolares en cada uno de los países.

Inicialmente concebido para los maestros especialistas de lengua inglesa, el Practicum Internacional está sirviendo en la actualidad, además, como una referencia valiosa para el diseño del nuevo Practicum en los grados de maestro en la USAL y Universidades de Castilla y León, al aportar no sólo el resultado de un diálogo entre lenguas y culturas diferentes, sino procedimientos novedosos, inspirados en protocolos de actuación previamente experimentados de manera efectiva con cohortes reducidas de estudiantes, para la supervisión y evaluación de las prácticas de alumnos de grado y de postgrado de ambos países que, además de los contenidos educativos, las destrezas pedagógicas y las actitudes profesionales, otorgan un lugar central a un factor de creciente relevancia en el nuevo perfil de los docentes europeos como es la competencia intercultural. De hecho "los tres grandes referentes escolares de la División de Política Lingüística de Estrasburgo -Marco (MCER), PEL (Portfolio Europeo Lingüístico) y Autobiografía de Encuentros Interculturales- son para todas las lenguas y no sólo para las lenguas extranjeras" (Vez, 2001, 105). Y así como hacemos nuestra a efectos operativos, antes de proseguir, la sencilla definición de cultura como "un conjunto de rasgos distintivos intelectuales, afectivos y emotivos, espirituales y materiales que caracterizan a aquellas personas que, con lenguas no necesariamente diferentes, pertenecen a entornos geográfi- 
cos, sociales y políticos diferentes" (Gonzalez, Guillén y Vez, 2010, 229), asumimos de forma congruente la competencia intercultural como "conjunto de conocimientos, destrezas, actitudes y comportamientos que [...] permiten a un hablante [...] reconocer, comprender, interpretar y, llegado el caso, aceptar otras maneras de entender la vida más allá de su propia cultura" (Gonzalez, Guillén y Vez, 2010, 230). Todavía más, al igual que el crítico del postcolonialismo Homi K. Bhabha (1990) ha desentrañado una continuidad indisociable entre las naciones y sus narraciones hasta el punto de considerar sus demarcaciones como umbrales de significado que han de ser cruzados en los procesos de producción cultural, nosotros podemos decir que, de modo semejante a las anteriores aunque extrapolando dicha vinculación al universo escolar, también los microrrelatos de los estudiantes de maestro -sus vivencias y sus testimonios-, al igual que el formato discursivo de este caso, deliberadamente narrativo, atraviesan fronteras pedagógicas y cosmovisiones culturales y trazan nuevos territorios de enseñanza, plasmaciones curriculares híbridas más complejas y portadoras de insospechada novedad y creatividad al poner en contacto tradiciones pedagógicas diversas que, al tiempo que nos interpelan, se fertilizan mutuamente (Davies y McKeon, 1993).

Así, al término de sus prácticas escolares, los estudiantes realizan una reflexión sobre las mismas en la forma de una autoevaluación acerca de su experiencia intercultural, vertebrada en torno a los siguientes ejes:

1. Interés en las formas de vida de otras personas.

2. Capacidad de cambiar de perspectiva.

3. Capacidad de afrontar el hecho de vivir en una cultura diferente.

4. Conocimiento sobre otro país y cultura.

5. Conocimiento sobre la comunicación intercultural.

A modo de ejemplo, Mónica F. (2010), una de las estudiantes, comentaba gratamente sorprendida que la familia con la que había estado residiendo durante las cuatro semanas de sus prácticas escolares en Nottingham era de origen indio y que había conocido fiestas tradicionales y probado recetas hasta entonces del todo desconocidas para ella.

Otra estudiante escribe en su diario de prácticas, redactado en su totalidad en lengua inglesa:

My experience at W. H. Primary school has been very positive and rich. It's not just having the teaching experience in the school but also the possibility of being immersed in all the aspects of the culture which has allowed me to learn important things. I've been placed in a school with children from very diverse social, cultural and economical backgrounds and a great number of disadvantaged children and a lot of children with Special Educational Needs. In the school, I was placed in one group, in year 5, which has been my "home" group during my placement. Being in one group has allowed me to have a deeper experience, as I've been able to learn about how to teach all the different areas of the English curriculum, and also to establish good relationships with many of the children in the classroom. My 
contribution to the classroom has consisted of first, teaching some Spanish through different activities like songs, tales, games, etc., and second, providing a support to the teacher in the rest of the areas of the curriculum, and I've worked particularly with those children who had Special Educational Needs. During my placement in the school in Nottingham, I've been able to learn about another Educational system and compare it with ours. These are the main differences I've noticed:

- In general, I've found that they use a more practical approach to learning. As they don't use textbooks as the main didactic resource as in Spain, the dynamics of the class gets another orientation, as they use more varied resources and activities.

- The new technologies are more integrated in the curriculum than in Spanish schools.

- They do lots of experimental activities that lead the children to get their own conclusions. All Science lessons I attended during my stay had this experimental approach.

- They spend a lot of time in reading activities, focusing on comprehension reading.

- The management of the classroom is also different. The teacher uses very different group dynamics in the classroom: individual work, pair work, group work, etc.

- The possibility of doing several activities in the classroom at the same time adapted to the different levels and abilities of the students, is the usual thing there. The curriculum is more adapted to the students' needs.

In conclusion, I think that having the chance of doing my teaching practice in two different cultural and educational contexts has given me the possibility of getting a new perspective about education (Cristina M., 2009).

Y el siguiente testimonio sintetiza en pocas palabras la riqueza, y a la vez complejidad, de los distintos escenarios educativos que en la actualidad son laboratorios de convivencia y multiculturalidad y un pequeño microcosmos de nuestro mundo:

Cuando me lo dijeron, y hasta que no lo vi con mis propios ojos, no me lo podía creer: 20 lenguas distintas en una clase de 25 alumnos, y los niños están felices y quieren ir al colegio y se entienden entre sí. Los maestros hacen una tarea extraordinaria, nada fácil, porque además, y sobre todo, han de limar las diferencias culturales entre los padres y hacerles partícipes del aprendizaje de sus hijos y explicarles lo que hacen para que puedan apoyar su tarea en casa. Tenemos mucho que aprender de ellos (Marta L., 2011).

Sus experiencias ofrecen amplias evidencias de que el Practicum Internacional supone para todos ellos un verdadero salto "extramuros" desde la vida del aula hasta el aula que es la vida. Sin duda, como observa Tomlinson $(2005,3)$, "lo que tenemos en común nos hace humanos. Lo que nos diferencia nos hace individuos".

Y una consecuencia de ello, en el ámbito escolar, es la siguiente:

La sociedad de la información exige una nueva concepción de la escuela, en tanto que ésta deja de ser una mera transmisora de conocimientos para convertirse en un espacio multicultural -hoy la normalidad está en la heterogeneidad, lo anómalo 
es la homogeneidad-, donde los distintos actores que interactúan "aprenden a aprender" unos de otros y unos con otros (Fernández, 2009, 22).

Hacemos, de ese modo, nuestras las reflexiones de Michael Byram, Bella Gribkova y Hugh Starkey (2002), quienes sostienen que el papel de los docentes de idiomas no se reduce al desarrollo exclusivo de capacidades lingüísticas. Además de éstas, y de manera ineludible, su tarea consiste en desarrollar destrezas, actitudes y valores al tiempo que facilitar el conocimiento de países o culturas específicas. Así, un perfil europeo para profesores de idiomas (Kelly, Grenfell, Allan, Kriza y McEvoy, 2004, 4-7) propone los siguientes 40 parámetros formativos bajo cuatro grandes apartados, que recogen todo lo anterior de forma sistematizada:

\section{STRUCTURE}

1. A curriculum that integrates academic study and the practical experience of teaching.

2. The flexible and modular delivery of initial and in-service education.

3. An explicit framework for teaching practice (stage/practicum).

4. Working with a mentor and understanding the value of mentoring.

5. Experience of an intercultural and multicultural environment.

6. Participation in links with partners abroad, including visits, exchanges or ICT links.

7. A period of work or study in a country or countries where the trainee's foreign language is spoken as native.

8. The opportunity to observe or participate in teaching in more than one country.

9. A European-level evaluation framework for initial and in-service teacher education programmes, enabling accreditation and mobility.

10. Continuous improvement of teaching skills as part of in-service education.

11. Ongoing education for teacher educators.

12. Training for school-based mentors in how to mentor.

13. Close links between trainees who are being educated to teach different languages.

\section{KNOWLEDGE AND UNDERSTANDING}

14. Training in language teaching methodologies, and in state-of-the-art classroom techniques and activities.

15. Training in the development of a critical and enquiring approach to teaching and learning.

16. Initial teacher education that includes a course in language proficiency and assesses trainees' linguistic competence.

17. Training in information and communication technology for pedagogical use in the classroom.

18. Training in information and communication technology for personal planning, organisation and resource discovery.

19. Training in the application of various assessment procedures and ways of recording learners' progress.

20. Training in the critical evaluation of nationally or regionally adopted curricula in terms of aims, objectives and outcomes.

21. Training in the theory and practice of internal and external programme evaluation. 


\section{STRATEGIES AND SKILLS}

22. Training in ways of adapting teaching approaches to the educational context and individual needs of learners.

23. Training in the critical evaluation, development and practical application of teaching materials and resources.

24. Training in methods of learning to learn.

25. Training in the development of reflective practice and self-evaluation.

26. Training in the development of independent language learning strategies.

27. Training in ways of maintaining and enhancing ongoing personal language competence.

28. Training in the practical application of curricula and syllabuses.

29. Training in peer observation and peer review.

30. Training in developing relationships with educational institutions in appropriate countries.

31. Training in action research.

32. Training in incorporating research into teaching.

33. Training in Content and Language Integrated Learning (CLIL).

34. Training in the use of the European Language Portfolio for self-evaluation.

\section{VALUES}

35. Training in social and cultural values.

36. Training in the diversity of languages and cultures.

37. Training in the importance of teaching and learning about foreign languages and cultures.

38. Training in teaching European citizenship.

39. Training in team-working, collaboration and networking, inside and outside the immediate school context.

40. Training in the importance of life-long learning.

Cada uno de ellos merecería una consideración específica y extensa, y de hecho todos son objeto de estudios discretos en el ámbito europeo, como puede verificarse consultando los recursos bibliográficos publicados por el Centro Europeo de Lenguas Modernas ${ }^{1}$.

Pues bien, consideramos que lo que se propone, justificadamente, para esta especialidad de la enseñanza primaria y secundaria, ocupa también un lugar de creciente importancia para cualquier educador que conciba la comunicación como una herramienta básica de su quehacer cotidiano y, con adaptaciones, resultaría igualmente útil como referencia orientativa para todo docente. Y es que en tiempos de crecientes flujos migratorios (Grinblat, 2010), de continua movilidad y de cambios vertiginosos en espacios complejos e interdependientes, la dimensión intercultural constituye en la actualidad el núcleo mismo de la escolaridad, pues dicha dimensión comprende: el desarrollo de destrezas interculturales, la preparación para la interacción con individuos y grupos sociales de otras culturas, la comprensión de perspectivas, valores y comportamientos diferentes, la superación

1. Página web de The European Centre for Modern Languages, disponible en: http://www. ecml.at/. 
de prejuicios y estereotipos y el aprecio de la diversidad como elemento de enriquecimiento humano y factor de democracia y madurez ciudadana. De hecho, tomar conciencia de la base cultural de las propias actitudes y comportamiento representa tanto para los jóvenes escolares como para los ciudadanos adultos la transformación de su mirada y el comienzo de la verdadera valoración de los otros en su diferencia (Merrill, 1986, 2). Suscribimos, por eso, el siguiente postulado que sitúa la interculturalidad como un factor de primer orden para la agenda educativa del presente siglo XXI:

Exponer la necesidad del carácter intrínsecamente intercultural de la acción educativa trae como consecuencias tanto una reformulación de la concepción tradicional en torno al aprendizaje de competencias culturales, formales o no formales, como la potenciación de la autocomprensión social y personal, permitiendo una construcción de la identidad cultural basada en el respeto, el reconocimiento y la valoración positiva de la pluralidad inherente al ser humano y la multiculturalidad presente en nuestras comunidades, de manera que la diferencia cultural deje de percibirse pedagógicamente como un déficit (Vila, 2005, 95).

Todavía más, existe una correlación entre la ética de y entre las culturas y la ética de y entre las personas. Nos hallamos ante una oportunidad única para que la razón instrumental se vea modulada por la razón que siente (Zubiri, 1980) y ambas operen de consuno, en el terreno colectivo, al servicio de una ética cordial, pues «encontrar esos valores que pueden compartir culturas diversas, en un diálogo emprendido desde ellas, es un desafío para una ética cívica, y difícil tendrá responder a él con entereza si no es ethica cordis" (Cortina, 2007, 27).

De manera sucinta, la enseñanza de idiomas y, por extensión, la enseñanza de la comunicación y la enseñanza como comunicación en sentido amplio quedan, en virtud de lo anterior, estrechamente vinculadas a las diferentes modalidades de "Saber" que acompañan a las competencias propiamente lingüísticas: a) saber ser (savoir être): curiosidad y apertura, disposición a suspender los juicios previos (esto es, los prejuicios) sobre culturas extranjeras o, lo que es igual, poder desplazar una posición autocentrada y adoptar, o comprender, puntos de vista diferentes; b) conocimiento (savoirs) de procesos sociales, de percepciones ajenas sobre la propia cultura y de datos, hechos e ideas sobre otros pueblos y culturas distintas y distantes entre sí, o diferentes pero cruzadas dentro de espacios geográficos y escenarios políticos comunes; c) capacidad de interpretar y relacionar (savoir comprendre): de "leer" documentos o acontecimientos de otras culturas, a fin de explicarlos y relacionarlos con los de la cultura propia; d) competencias en los campos del descubrimiento e interacción (savoir apprendre/faire): adquisición de conocimientos nuevos sobre una nueva cultura y sus prácticas de convivencia y comunicación; y e) desarrollo de la sensibilidad y discernimiento críticos (savoir s'engager) a fin de evaluar de manera crítica, y sobre la base de criterios explícitos, perspectivas, prácticas y productos de la propia cultura o de culturas extranjeras (Council of Europe 2001, 101-106). 
Al formular el aprendizaje en términos de "saberes", y no sólo de conocimientos, se abre la posibilidad de que los diseños didácticos hagan suya, atravesándolos, una dimensión cualitativa que de otra forma podría ignorarse al hacer una lectura reduccionista, exclusivamente instrumental, de las competencias educativas. Para establecer una primera distinción somera viene en nuestra ayuda, de manera oportuna, la conclusión de un occidental tras un deliberado desarraigo cultural provisional con el que fue capaz de "arraigar" posteriormente su aprendizaje oriental en su lugar de origen:

Sólo se sabe lo que se experimenta. La información [...] sólo puede resultar de gran ayuda en el análisis y asimilación de nuestras propias vivencias, pero no es, en sí misma, una fuente de sabiduría [... E El conocimiento es la esencia que las facultades superiores de la mente libran en cada experiencia [...] Sin experiencia no hay auténtico conocimiento, y sin éste, no hay libertad posible (López-Seivane, 2007, 25-26).

Hasta tal punto resulta relevante la competencia intercultural en la Europa que aspira a ser de los ciudadanos, y no sólo de los mercaderes, que el Partnership Programme on European Youth Worker Training, dirigido por la Comisión Europea y el Consejo de Europa, ha publicado un "kit de formación" dirigido a jóvenes trabajadores y a formadores de jóvenes como una herramienta teórico-práctica fruto del esfuerzo colectivo de personas y organizaciones de muy distinto perfil -educadores, líderes de ONG y escritores profesionales- en el que se abordan conceptos relacionados con el aprendizaje intercultural y los valores en Europa y se ofrecen propuestas didácticas en forma de dinámicas de grupo, debates, juegos de simulación y de rol, investigaciones y presentaciones, además de talleres formativos (Martinelli y Taylor, 2010).

Podemos afirmar que con estas o similares propuestas formativas, como talleres de cross-cultural awareness (Odeniyi y Lazar, 2011, 2-8) de carácter vivencial y activo, la motivación de los participantes tiende a incrementarse, se genera cohesión, se estrechan vínculos y se estimula la participación y el compromiso colaborativo, es decir, que el empleo de instrumentos pedagógicos de trabajo dinámico, a la vez "implicado" y aplicado, potencia el desarrollo eficaz del denominado feedforward (Goldsmith, 2006), que permite a los agentes partir de su realidad concreta (inquietudes, aspiraciones, dudas, emociones, etcétera) al tiempo que les empuja hacia un futuro abierto a un abanico de posibles soluciones de diversa índole para una misma situación problemática, anticipando oportunidades constructivas y resultados positivos desde la atalaya de su realidad presente allí donde antes no se hubieran podido concebir. De ese modo, se supera la pretensión de exclusividad de la evaluación retrospectiva (feedback) y se generan las condiciones para logros de aprendizaje prospectivos, lo que permite educar el empeño por la transformación personal y profesional como "arte de la posibilidad" (Zander y Zander, 2000). 
Bajo esa luz, empresas educativas como el Practicum Internacional, conducentes a compartir experiencias de formación y a tender puentes entre la reflexión y la práctica, entre la lengua y la cultura, entre educadores y estudiantes de diferentes países, se vuelven no sólo aconsejables sino fundamentales, y sus frutos hacen factible su generalización. Si la competencia intercultural supone una praxis susceptible de vertebrar la escolarización desde sus etapas más tempranas (Rábano, 2010; Aguado, 2010; Pedraz, 2009; Buitrago, 2009; Vez y Piñeiro, 2004) tanta mayor importancia adquiere como elemento fundamental en la formación docente y como el quicio o el interfaz entre la teoría aplicada y la práctica pensada que es el Practicum.

En el caso de la USAL hay que decir que, previo al convenio de colaboración suscrito con el MEC en el curso 2004-2005, la Escuela Universitaria de Ávila y las Facultades de Educación y Departamento de Idiomas Modernos de la NTU así como las Autoridades Educativas Locales (LEA) de Nottingham (County y City Council), ya habían venido desarrollando durante diez años programas diversos de cooperación de manera regular, bien que de modo voluntario y sin cauces institucionales arbitrados a ese efecto. El elenco de vínculos previos había venido conformando, pues, un tejido constructivo favorecedor de un clima amistoso de colaboración, lo que preparó el terreno para una comunicación fluida, una coordinación satisfactoria y una consolidación de experiencias previas y exitosas con cauces esta vez más formales. No es tan sólo anecdótico, en consecuencia, sugerir que el criterio evaluable de calidad institucional vino fundamentado por un presupuesto previo de calidez interpersonal, que siguió informando de manera decisiva cada nueva edición y permitió afrontar con ecuanimidad tanto los logros como las dificultades, de las que tampoco se ha visto exenta esta apuesta pedagógica transfronteriza y transdisciplinar. El nuevo marco institucional favoreció, no obstante lo anterior, cambios cualitativos importantes y logró incentivar de manera extraordinaria a los estudiantes que vieron respaldadas económicamente sus aspiraciones a la vez que, también por primera vez, permitió acoger a estudiantes de postgrado (PGCE) de Nottingham y facilitar igualmente para ellos las prácticas en los Campus de Zamora y Ávila de la USAL.

La iniciativa de intercambio de un promedio de diez estudiantes anualmente de cada país en su periodo de prácticas escolares, supervisados por maestros y profesores universitarios durante cuatro semanas, se inscribe en dos grandes marcos u horizontes de sentido, dentro de los cuales cabe entender su máxima relevancia, a saber: a) la necesidad imperiosa, de orden global, de acercar pueblos y culturas combatiendo procesos de confrontación y "choque", actitudes de xenofobia o intolerancia y fomentando el conocimiento mutuo y la cooperación en tareas constructivas y favorecedoras del aprecio recíproco de las personas, los pueblos y sus culturas desde la primera infancia (Claire, 2006); y b) el actual proceso de convergencia europea en el ámbito de la educación superior, que obliga a los agentes educativos a buscar estrategias universitarias conjuntas, algo singularmente necesario en el terreno específico de la formación del profesorado de enseñanza 
primaria y secundaria, y en "la cooperación internacional en el campo de las lenguas modernas" (Morrow, 2004, 13).

Así pues, se puede decir que en cierto modo se fueron materializando progresiva y felizmente, en lo pequeño, aquellos importantes cambios que la demanda social y que los gobiernos e instituciones propician a gran escala. Para sugerir un nuevo correlato entre la pequeña constelación formativa en la que queda circunscrito este proyecto y el crisol de culturas sin precedentes que hoy en día es nuestro mundo, resulta iluminadora la propuesta del Premio Nobel contemporáneo Amartya Sen:

The main hope of harmony in our troubled world lies in the plurality of our identities, which cut across each other and work against sharp divisions around one single hardened line of vehement division that allegedly cannot be resisted. Our shared humanity gets savagely challenged when our differences are narrowed into one devised system of uniquely powerful categorization (2006, 16-17).

El desarrollo organizativo y los frutos derivados de este convenio desde el curso académico 2004-2005 han quedado reflejados en informes periódicos elevados al Servicio de Relaciones Internacionales de la USAL y, desde éste, al Instituto de Formación del Profesorado del Ministerio de Educación y a la TDA (Training and Development Agency for schools) del Departamento de Educación británico así como en tres proyectos de innovación educativa ${ }^{2}$. Una síntesis de los mismos permite distinguir cinco fases en su desarrollo:

1. La primera de ellas se remonta al Memorándum de entendimiento entre el Ministerio de Educación, Cultura y Deporte con el Departamento de Educación británico con el objetivo de potenciar la cooperación conjunta en el campo de formación inicial del profesorado y el desarrollo de lenguas extranjeras en la educación primaria, con aplicación a partir de noviembre de 2003.

2. Por lo que respecta al convenio de colaboración entre la NTU y la USAL, tras unos tanteos iniciales para determinar la disposición de ambas

2. Beltrán, F.; Durán, R. y SÁnchez-Reyes, S. (2010) Adaptación de asignaturas troncales de la actual titulación de maestro especialista en lengua inglesa a las materias y mención de lengua inglesa en los grados de maestro de educación infantily primaria (ID9/200). Ayudas de la Universidad de Salamanca para la innovación docente, curso 2009-2010. http://gredos.usal.es/jspui/handle/10366/81783; DuRÁN, R.; Beltrán, F. y SÁnChez-Reyes, S. (2010) Diseño y utilización de materiales audiovisuales a través de Studium basados en prácticas docentes en el Reino Unido para los grados de Maestro en Educación Primaria e Infantil (ID9/201). Ayudas de la Universidad de Salamanca para la innovación docente, curso 2009-2010. http://gredos.usal.es/jspui/handle/10366/81784; Alonso, P.; GutiérreZ, G.; Durán, R. y Beltrán, F. (2006) Diseño de un Practicum europeo para la formación del profesorado en lengua inglesa. Proyectos en torno a la convergencia europea de la enseñanza en las universidades de Castilla y León. Junta de Castilla y León (Orden EDU/995/2005, de 19 de julio de 2005 y concesión de ayuda en BOCYL, n. ${ }^{\circ} 234$, de 5 de diciembre de 2005). 
universidades, los primeros encuentros de coordinación entre la representante de la NTU y las Direcciones de las Escuelas Universitarias tuvieron lugar en el mes de junio de 2004.

3. A éstos les siguió una primera convocatoria oficial por parte del Ministerio de Educación y Ciencia en Madrid, el día 28 de octubre de 2004, en la que ya se abordaron cuestiones específicas de la coordinación relativas a las figuras de los coordinadores y definición de sus tareas, fechas de realización de las prácticas, número de estudiantes, alojamiento, financiación, procesos de selección de estudiantes y de centros, calendario de tareas para los estudiantes y de visitas de tutorización y supervisión, protocolos de observación y evaluación, etc.

4. A partir de ese momento se inició un periodo intenso de contactos con el Servicio de Relaciones Internacionales del Vicerrectorado de Relaciones Institucionales de la USAL, en noviembre de 2004, para abordar cuestiones logísticas tales como la convocatoria de las becas, la gestión de alojamientos, tramitación de pagos, seguros de asistencia sanitaria y justificaciones finales de actividades.

5. Desde ese momento, y con el pleno conocimiento y apoyo de las comisiones de prácticas de los dos centros de la USAL y los responsables de la NTU, se abrió una etapa de comunicaciones continuas entre todas las partes:

- Coordinadores de la USAL entre sí y con el Servicio de Relaciones Institucionales.

- Coordinadores de la USAL y NTU entre sí y con el Servicio de Relaciones Institucionales de la USAL y su par en la NTU.

- Coordinadores de la USAL y de la NTU con sus estudiantes, con los maestros y tutores, y con los anfitriones, familias o responsables de las Residencias de los estudiantes de ambos países.

- $\quad$ Estudiantes de ambos países y universidades entre sí.

Y todo ello se efectuó a través de encuentros personales, visitas al efecto, contactos telefónicos, correos electrónicos o ágil envío de documentos por parte del Servicio de Relaciones Internacionales de la USAL. Esa densidad de comunicación permitió efectuar las tareas propias de gestión, tutoría y orientación educativa, supervisión y evaluación según los parámetros acordados entre los coordinadores de ambas universidades con conocimiento pleno del Servicio de Relaciones Internacionales de la USAL y sus pares institucionales en la NTU. Si describimos estas cuestiones organizativas es porque forman parte del tejido de relaciones que, de forma paralela a la administración institucional, ha ido acercando a las personas a través de sus diferentes cometidos hacia objetivos de interés común, que han tenido que ir haciéndose más explícitos.

En este punto, y en lo que respecta a los contenidos propiamente del periodo de prácticas escolares, hay que decir que las universidades británicas, francesas, alemanas, italianas y españolas acordaron un modelo espiral alrededor 
de cuatro coordenadas de competencias (profesional, pedagógica, lingüística e intercultural), con diez objetivos que amplían su alcance y profundidad de forma progresiva en el transcurso de las prácticas escolares alrededor de tres áreas transversales (observación, implementación y evaluación). La espiral relativa a la competencia intercultural constituye, por tanto, un elemento axial del nuevo "mapa" y ciertamente del territorio educativo europeo:

\section{GRÁFICO 1. COMPETENCIA INTERCULTURAL}

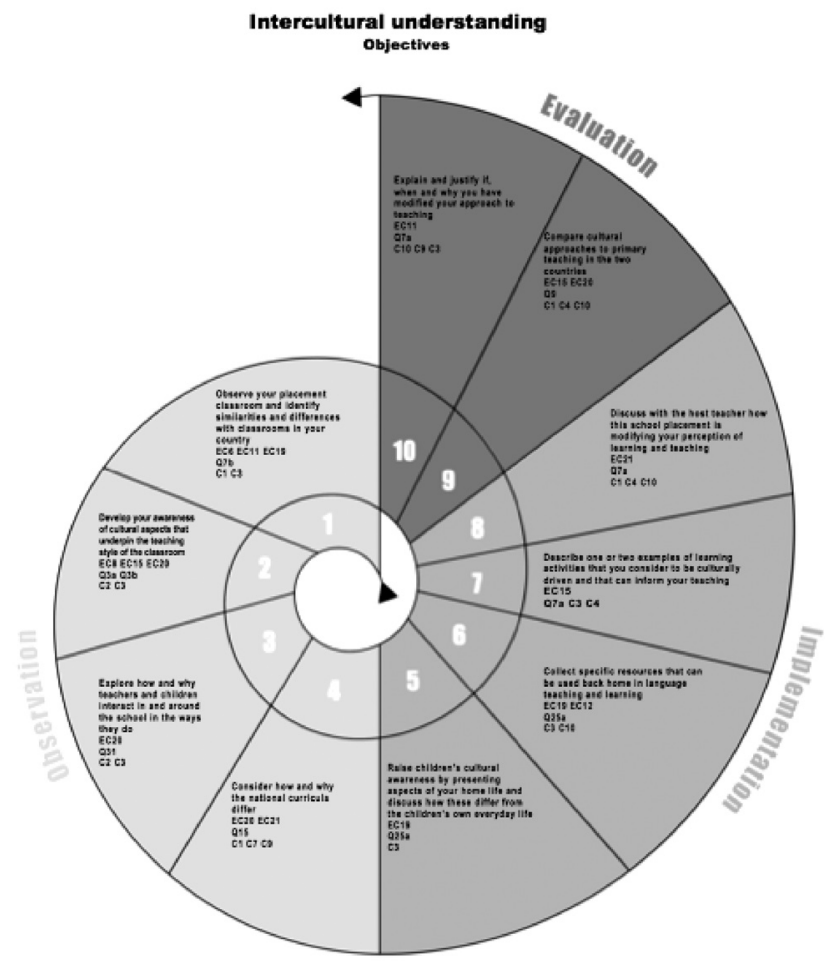

Fuente: The Common Reference Framework. Department for children, schools and families/ Training and development agency for schools ${ }^{3}$.

3. Un borrador completo de la versión bilingüe en inglés-español de fecha 25/02/2010, se puede descargar en formato pdf en la siguiente página web: http://www.tda.gov.uk/training-provider/ itt/developing-quality/language-exchanges/common-reference-framework.aspx. Consultado el $12 \mathrm{de}$ julio de 2011. 
A cada objetivo le sigue una clave de equivalencias entre paréntesis, de la que ofrecemos tan sólo la primera a título de ilustración:

$\mathrm{CE}+$ número $>$ Competencias del profesorado europeo

Q + número > Criterios ingleses para la titulación de profesor cualificado (Qualified Teacher Status)

CES + número > Competencias españolas del Grado de Maestro

\section{CuAdro 1. Objetivos DE la COMPETEnCIA InTERCUltural}

\begin{tabular}{|c|c|c|}
\hline \multirow{3}{*}{ 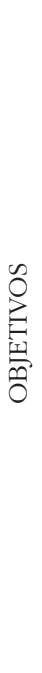 } & Observación & $\begin{array}{l}\text { 1. Observar la diversidad cultural en el aula y en el centro (CE1-Q7B-CES3) } \\
\text { 2. Analizar la respuesta educativa a la diversidad en el aula } \\
\text { 3. Describir uno o dos ejemplos de actividades educativas que considere } \\
\text { que tienen un fuerte componente intercultural y que pueden ser } \\
\text { enriquecedoras para su formación } \\
\text { 4. Reflexionar con el TC (Tutor del Centro) sobre la atención a la diversidad } \\
\text { cultural y cómo utilizarla para enriquecer el aprendizaje }\end{array}$ \\
\hline & Implementación & $\begin{array}{l}\text { 5. Fomentar el conocimiento cultural de los alumnos mediante la presentación } \\
\text { de situaciones reales en la vida cotidiana de su país de origen y comentar } \\
\text { con ellos el modo en que éstas difieren de las suyas propias } \\
\text { 6. Diseñar la planificación docente incorporando la perspectiva de la } \\
\text { diversidad } \\
\text { 7. Desarrollar actividades educativas que considere que fomentan la } \\
\text { interculturalidad en el aula } \\
\text { 8. Contrastar los enfoques culturales de la enseñanza primaria en los dos } \\
\text { países }\end{array}$ \\
\hline & Evaluación & $\begin{array}{l}\text { 9. Comentar con el TC cómo están modificando estas prácticas docentes, su } \\
\text { percepción de la enseñanza y el aprendizaje } \\
\text { 10. Valorar y explicitar cómo se ha modificado su práctica docente a través } \\
\text { de este proceso }\end{array}$ \\
\hline
\end{tabular}

Debe añadirse que al término de cada una de las ediciones de las prácticas escolares ha habido reuniones con los estudiantes y comunicaciones ulteriores entre los coordinadores al objeto de evaluar la experiencia. Los estudiantes británicos, por su parte, culminan su estancia con la entrega de los portafolios de documentación requeridos en su esquema de Teaching Practice y los españoles con la elaboración de un Teaching Practice Report, esto es, un informe o memoria de prácticas, en el que vierten de manera articulada elementos descriptivos y reflexivos de su periodo de inserción escolar a partir de pautas detalladas para su redacción que se proporcionan a los estudiantes en un documento diseñado al efecto y que incluyen, entre otros, aspectos tales como:

- El colegio y el entorno escolar.

- El aula: información contextual sobre la clase; características del grupo; el tutor de la clase (y otros maestros que den clase a ese grupo); otras personas que participan en el aula: ayudantes, padres, etc.; la coordinación entre los diferentes profesores y materias; la organización de la clase 
(agrupamientos, flexibilidad, formas corrientes de interacción, disciplina, etc.); niños con características/necesidades especiales; la evaluación y la disciplina.

- Las segundas lenguas (L2) en el currículum escolar; el papel de las L2 en el colegio; metodología; la coordinación del profesor de L2 con otros maestros/áreas curriculares; los materiales y recursos para la enseñanza del Inglés como Lengua Extranjera; las historias, canciones, juegos, actividades, proyectos, festivales, acontecimientos especiales, etc., de interés para la enseñanza del inglés como L2.

- Las formas de obtener una Cualificación como Profesor (Qualified Teacher Status) y posibilidades de conseguir un trabajo en el campo de la enseñanza en el Reino Unido.

Si bien originalmente concebido para facilitar una experiencia de cooperación internacional cuyos beneficiarios principales habrían de ser los aprendices de maestro, estudiantes de magisterio o teacher trainees, lo cierto es que también los maestros de los colegios en España y el Reino Unido así como los coordinadores universitarios hemos iniciado un capítulo nuevo de aprendizaje al vernos obligados a clarificar posturas, a definir metas y objetivos formativos y a articular de modo congruente las propuestas organizativas de las prácticas escolares, así como a acordar y a explicar esquemas de evaluación o a sistematizar siquiera mínimamente protocolos de observación e informes "de campo" durante las observaciones de los trainees. Lejos de ser estéril, o de provocar cansancio o desaliento, ese cúmulo de pequeños esfuerzos comunicativos combinados ha tenido, al menos, dos efectos sumamente positivos: por un lado, demostrar que es posible desarrollar elaborados programas educativos de manera constructiva en el ámbito internacional y, por otro, constatar que resulta muy enriquecedor contrastar experiencias educativas diversas desde la enseñanza primaria hasta la universitaria y aprender de las similitudes y diferencias entre todas ellas.

Las espontáneas expresiones de alegría de los niños de los colegios británicos y de los españoles por contar con nuevas presencias adultas en el aula, y los niveles de participación, empatía y aprecio de sus tutoras proporcionan argumentos sólidos para acreditar nuestras apreciaciones. No es, a este respecto, ajena a nuestra propia mirada educativa "la convicción cultural de que es posible planificar la intervención formativa, teniendo en cuenta el estrato emocional y que, de hecho, esa intervención ha constituido una parte importante de las comunidades culturales a lo largo de la historia" (García Carrasco, 2006, 28).

Adicionalmente, la oportunidad privilegiada de hablar de forma extensa con los estudiantes de ambos países para nosotros ha comportado un nuevo desafío ya que, de manera muy sincera y avalada por su experiencia, han puesto el dedo en la llaga en temas educativos de enorme importancia obligándonos a repensar numerosos aspectos sobre la teoría y la práctica de la enseñanza de los contenidos curriculares en los colegios, entre los que destacan, sin quedar limitados a ellos, los referidos a la enseñanza de las lenguas extranjeras. Así, a través de sus 
comentarios, formales e informales, han señalado interrogantes respecto a multitud de cuestiones relativas a métodos de enseñanza centrados en el alumno o en el profesor; o respecto al uso o al abuso de los libros de texto; a la prioridad o difícil equilibrio entre los componentes de socialización y los de los conocimientos en los procesos de aprendizaje; a los niveles de coordinación entre los profesores, a los códigos de cortesía obligados entre los escolares, sus maestros y los padres o tutores; o respecto a la determinación familiar, social, económica o incluso étnica a la hora de favorecer o frustrar el éxito escolar, y un largo etcétera en el que tienen cabida, por supuesto, diferencias culturales de horarios, hábitos alimenticios, modas, modelos de convivencia familiar, cuestiones de género, fenómenos sociales tales como el bullying, mobbing, etc.

De forma extraordinariamente motivadora para los estudiantes resulta, por supuesto, el progreso lingüístico experimentado en tan sólo cuatro semanas de exposición intensa a la lengua inglesa e inmersión en entornos angloparlantes, especialmente concentrados en las aulas, donde el lenguaje de las instrucciones tiene tanta importancia como el propio contenido. La siguiente muestra, recogida por una estudiante a lo largo de sus cuatro semanas de inmersión en un colegio de Nottingham en respuesta a una de las tareas que tienen que realizar, donde precisamente se les pide que hagan una compilación y categorización del llamado classroom language - para diferenciarlo del subject content vocabulary-, fue además objeto de aplicación posterior en sesiones de "microenseñanza" en las aulas de Magisterio y en los colegios españoles, y da cuenta del amplio espectro de interacciones discursivas y performativas en el aula:

\section{CuAdro 2. Classroom language}

\begin{tabular}{|c|c|}
\hline Greetings & $\begin{array}{l}\text { - Good morning, everybody. } \\
\text { - Good afternoon, everybody. } \\
\text { - Hello, everyone. } \\
\text { - Hi there! } \\
\text { - How are you today? } \\
\text { - How are things with you? } \\
\text { - Are you feeling better today? }\end{array}$ \\
\hline Time to begin & $\begin{array}{l}\text { - Let's begin our lesson now. } \\
\text { - Is everybody ready to start? } \\
\text { - Get ready to listen to your instructions. } \\
\text { - All right then. } \\
\text { - Ok, everyone! } \\
\text { - Listen! } \\
\text { - I'm waiting for you to be quiet. } \\
\text { - We won't start until everybody is quiet. } \\
\text { - Face the teacher, please! }\end{array}$ \\
\hline Register & $\begin{array}{l}\text { - All right, then, I'll take the register first! } \\
\text { - Who is absent today? } \\
\text { - Good morning/afternoon, Miss Bezzano. } \\
\text { - Sandwiches/dinners, please! }\end{array}$ \\
\hline
\end{tabular}




\begin{tabular}{|c|c|}
\hline $\begin{array}{c}\text { Simple } \\
\text { instructions }\end{array}$ & $\begin{array}{l}\text { - } \text { Come in. } \\
\text { - Go out. } \\
\text { - Stand up. } \\
\text { - Sit down. } \\
\text { - Come to the front of the class. } \\
\text { - Stand by your desks. } \\
\text { - Put your hands down. } \\
\text { - Hold your books/pens up. } \\
\text { - Show me... } \\
\text { - You need your pencils/rulers/little whiteboards... } \\
\text { - } \text { (student's name), come and sit down on the carpet, please! } \\
\text { - Tray inspection now! } \\
\text { - We'll learn how to... } \\
\text { - Can you tell me a proper sentence? } \\
\text { - Has anybody got any idea about...? } \\
\text { - Put the announcements into your drawers. } \\
\text { - A few seconds to think about good things about... } \\
\text { - Are you ready? } \\
\text { - Open your books at page... } \\
\text { - Turn to page... } \\
\text { - Look at activity five. } \\
\text { - You have five minutes to do this. } \\
\text { - Who's next? } \\
\text { - Like this, not like that. } \\
\text { - It's time to finish. } \\
\text { - Have you finished? } \\
\text { - Let's stop now. } \\
\text { - Let's check the answers. } \\
\text { - Any questions? } \\
\text { - } \text { - Back your desks tidy? } \\
\text { - Put down your pens and pencils. } \\
\end{array}$ \\
\hline $\begin{array}{c}\text { Comprehension } \\
\text { language }\end{array}$ & $\begin{array}{l}\text { - OK so far? } \\
\text { - Do you get it? } \\
\text { - Do you understand? } \\
\text { - Do you follow me? } \\
\text { - Say it again, please. } \\
\text { - Is that clear to everybody? } \\
\text { - I don't understand. } \\
\text { - I don't get it. } \\
\text { - Like this? } \\
\text { - Is this OK? } \\
\text { - I need some help. }\end{array}$ \\
\hline Time to stop & $\begin{array}{l}\text { - It's almost time to stop. } \\
\text { - I'm afraid it's time to finish now. } \\
\text { - We'll have to stop here. } \\
\text { - That's all for today. You can go now. } \\
\text { - We'll finish this exercise next lesson. } \\
\text { - We've run out of time, so we'll continue next lesson. }\end{array}$ \\
\hline
\end{tabular}




\begin{tabular}{|c|c|}
\hline $\begin{array}{l}\text { Asking the } \\
\text { students to wait }\end{array}$ & $\begin{array}{l}\text { - Hang on a moment. } \\
\text { - Just hold on a moment. } \\
\text { - Stay where you are for a moment. } \\
\text { - Just a moment, please. }\end{array}$ \\
\hline Saying good bye & $\begin{array}{l}\text { - Goodbye, everyone. } \\
\text { - See you tomorrow. } \\
\text { - See you after the break. } \\
\text { - Have a good weekend/holiday } \\
\text { - Enjoy your holiday. }\end{array}$ \\
\hline $\begin{array}{l}\text { Leaving the } \\
\text { classroom }\end{array}$ & $\begin{array}{l}\text { - Line up! } \\
\text { - Try not to make any noise as you leave! }\end{array}$ \\
\hline $\begin{array}{l}\text { Classroom } \\
\text { management }\end{array}$ & $\begin{array}{l}\text { - Make groups of four. } \\
\text { - Move your desks into groups of four people. } \\
\text { - Find a partner. } \\
\text { - What group are you in? } \\
\text { - Work in pairs/threes/fours/fives. } \\
\text { - Work in groups of two/three/four. } \\
\text { - I want you to form groups. } \\
\text { - Form groups of three. } \\
\text { - Can you join the other group? } \\
\text { - Only three people in each group. } \\
\text { - Everybody work individually. } \\
\text { - Work by yourselves. } \\
\text { - Work independently. } \\
\text { - Ask your neighbour for help. } \\
\text { - Work on the task together. } \\
\text { - Have you finished? } \\
\text { - Do the next activity. } \\
\text { - Move on to the next activity. } \\
\text { - Come out and write it on the board. } \\
\text { - Look around and say examples of... } \\
\text { - Show me... } \\
\text { - I'd like to ask (student's name) to come. } \\
\text { - Find a seat with your talk partner } \\
\text { - Let's sing a song. } \\
\text { - All together now. } \\
\text { - I want you all to join in. } \\
\text { - Could you try the next one? } \\
\text { - I would like you to write this down. } \\
\text { - Who would like to read? }\end{array}$ \\
\hline Sequencing & $\begin{array}{l}\text { - First of all, today... } \\
\text { - Right. Now we will go on to the next... } \\
\text { - What I want you to do now is... } \\
\text { - For the last thing today, let's... } \\
\text { - Whose turn is it to read? } \\
\text { - Which question are you on? } \\
\text { - Ready for the next instruction? } \\
\text { - Next one, please. } \\
\text { - Who hasn't answered yet? } \\
\text { - The idea of this exercise is for you to... } \\
\text { - You have ten minutes to do this. } \\
\text { - Finish this by twenty to eleven. } \\
\end{array}$ \\
\hline
\end{tabular}




\begin{tabular}{|c|c|}
\hline Giving feeback & 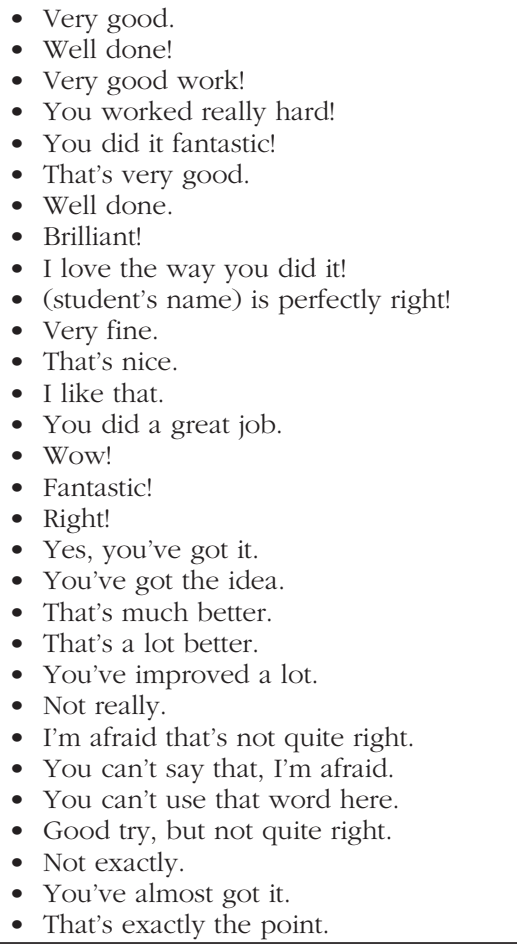 \\
\hline $\begin{array}{l}\text { Demanding } \\
\text { good behaviour }\end{array}$ & $\begin{array}{l}\text { - Sit down properly! } \\
\text { - You shouldn't be up! } \\
\text { - Don't swing on your chair! } \\
\text { - It's not acceptable to... } \\
\text { - Do you know the rule? } \\
\text { - I'm going to insist on... } \\
\text { - You can't use... } \\
\text { - I'm demanding silence! } \\
\text { - The way you behave is not acceptable. } \\
\text { - Stop it! } \\
\text { - You need to be listening! } \\
\text { - You failed to do that. } \\
\text { - There's no point making noises. } \\
\text { - Stop talking and be quiet. }\end{array}$ \\
\hline
\end{tabular}

El hecho de participar en la enseñanza de otras materias en inglés permite a los estudiantes apreciar los riesgos y oportunidades potenciales del enfoque de enseñanza que hace de la lengua inglesa una herramienta comunicativa y el vehículo para la instrucción de otras materias (Science, Arts and crafts, etc.), una fórmula cada vez más generalizada en Europa (EURYDICE, 2006, 2010) y cuyo proyecto pionero en España, iniciado en el año 1996 entre el Ministerio de Educación 
y el British Council, ha sido recientemente evaluado para satisfacer tres objetivos: 1) Proporcionar información basada en la investigación sobre la competencia lingüística de los alumnos en inglés, desarrollada y demostrada a través del estudio de varias asignaturas en un contexto bilingüe, así como sus progresos en español; 2) identificar y difundir las buenas prácticas que se den en los centros educativos participantes en el programa; y 3) proporcionar datos obtenidos mediante investigación sobre la sensibilización, actitud y motivación de los participantes en el programa. El programa de educación bilingüe en España dedica un porcentaje significativo del tiempo curricular a la enseñanza en la lengua adicional (en este caso, el inglés) que supone aproximadamente el 40\% del tiempo lectivo semanal, lo que permite seguir en inglés diversas asignaturas e introduce de forma temprana el aprendizaje de la lectoescritura en lengua inglesa, con el fin de complementar las habilidades de comprensión y expresión oral así como de promover una buena competencia lingüística subyacente en la lengua adicional (Dobson, Pérez y Johnstone, 2010). Es de destacar que esta iniciativa, que ya cuenta con 15 años de recorrido, está siendo objeto de la mayor atención en la actualidad tanto por la creciente generalización de los programas integrados en colegios de titularidad pública, concertada y privada como por la exigencia para todos los estudiantes de los nuevos Grados de Maestro de Enseñanza Primaria e Infantil, y no sólo para quienes escojan la Mención de Lengua Extranjera, de "expresarse en alguna lengua extranjera según el nivel B1, de acuerdo con el Marco Común Europeo de Referencia para las Lenguas" ${ }^{4}$.

\section{CONClusiones}

Para todos los participantes del Practicum Internacional, la experiencia humana de haber convivido, compartiendo tiempos de ocio y de trabajo fuera de su ambiente familiar ha significado la ampliación de sus miras, una mayor estima de sus posibilidades y una firme confianza en el hecho de poder transitar por el mundo, dialogar con él y hacer una contribución inestimable al mismo desde su atalaya profesional. Han descubierto, en suma, que su identidad no es algo únicamente recibido de forma pasiva, sino una construcción activa, una promesa y un compromiso personal continuo. Su experiencia forma parte de una educación triplemente integradora, esto es, orientada a la persona entera, capaz de reconocer la riqueza

4. Orden ECI/3857/2007, de 27 de diciembre, por la que se establecen los requisitos para la verificación de los Títulos Universitarios oficiales que habiliten para el ejercicio de la profesión de Maestro en Educación Primaria ( $B O E$ n. ${ }^{\circ} 312$, de 29 de diciembre de 2007).

Orden ECI/3854/2007, de 27 de diciembre, por la que se establecen los requisitos para la verificación de los Títulos Universitarios oficiales que habiliten para el ejercicio de la profesión de Maestro en Educación Infantil (BOE n. ${ }^{\circ} 312$, de 29 de diciembre de 2007). 
de la diversidad cultural planetaria, y promotora de un equilibrio efectivo entre la teoría y la práctica (Casassus, 2004, 15-16).

Por supuesto, jamás se trata de un recorrido concluso, ni solamente exitoso o exento de aspectos susceptibles de mejora, pero esta experiencia representa ni más ni menos que la meta y el sentido auténtico del Practicum Internacional por constituir un puente entre culturas y una herramienta para el auténtico entendimiento interpersonal e intercultural, cuyo test de validación consiste, precisamente, parafraseando a Austin (1982), en "hacer cosas [juntos] con palabras". A la capacidad intrínseca de los idiomas para comunicar, crear entendimiento y cruzar fronteras, se suma, pues, la rica posibilidad de todos los educadores de tender puentes: entre niveles educativos (enseñanza primaria y universitaria); entre instituciones locales, nacionales e internacionales (colegios, centros de formación de profesores, universidades, autoridades educativas); entre disciplinas diversas (como apunta el llamado enfoque AICLE o de Aprendizaje Integrado de Contenidos y Lenguas Extranjeras); entre culturas (y dentro de ellas, entre culturas pedagógicas diversas); y entre los estudiantes de distintas promociones para compartir sus experiencias y desarrollar nuevos proyectos de colaboración y entrañar lo que inicialmente fuera cultural y pedagógicamente extraño. De hecho, como concluye uno de los profesores participantes en el Practicum Internacional en un estudio sobre la experiencia intercultural de los estudiantes de maestro españoles,

the responsibility for effective exposure to relevant intercultural experiences in teacher training needs to be considered as a shared objective both by the host and the guest institutions -fostering this or similar stay abroad visits, links or exchanges, beneficial to their educational agendas- and by the individual students who need to integrate these personal, cultural, educational and linguistic challenges within their experiential portfolio and professional profile (Durán, 2011, 129).

Esas conclusiones se corresponden de manera general -en lo que respecta a la perspectiva de los educadores universitarios- con las de un estudio similar realizado desde la parte británica, bien que con matices distintivos derivados precisamente de los diferentes itinerarios formativos en España y el Reino Unido. En el mismo se destaca que entre los beneficios más notables de la experiencia del Practicum Internacional por parte de los estudiantes ingleses se contaban el aumento de su confianza en el uso de la lengua y una mayor comprensión intercultural (Woodgate-Jones, 2008, 8).

Se trata, en suma, de un prometedor punto de partida, que ha dado múltiples frutos, pero no de un punto de llegada. Y es que, en momentos de cambios vertiginosos como los que atravesamos, además de los múltiples logros que se derivan de unas prácticas internacionales, se hace necesario señalar numerosos retos pendientes que, si bien no exentos de obstáculos, suponen nuevos acicates para la acción, para la reflexión y para el compromiso docente. Algunas de esas tareas que hay que acometer son: hacer de la experiencia comunicativa (potencialmente plurilingüe) e intercultural un componente central de la formación de todos los 
maestros; fomentar el papel de los estudiantes universitarios como verdaderos embajadores a la vez que actores culturales; promover crecientes vínculos entre las escuelas anfitrionas de los países colaboradores mediante intercambios de materiales, videoconferencias, correspondencia electrónica, visitas, etc.; favorecer el "hermanamiento" formal de los colegios a fin de contribuir a crear, desde el ámbito escolar, una mayor y mejor ciudadanía europea; alentar el diseño de programas escolares concertados entre colegios de prácticas de ambos países; extender no sólo los programas de enseñanzas integradas, CLIL (Content and Language Integrated Learning) o content-based en los idiomas y entre colegios de distintos países, sino también los estudios sobre su eficacia y factores distorsionadores (Ruiz de Zarobe y Jiménez, 2009; Lasagabaster y Ruiz de Zarobe, 2010); ampliar la red de vínculos con otros colegios de enseñanza primaria y secundaria más allá del programa específico del Practicum Internacional mediante programas Comenius, e-twinnings o similares. Y, con las anteriores, en el área de formación del profesorado: desarrollar de manera conjunta módulos de enseñanza escolar, diseñar y llevar a cabo programas de formación internacional inicial y continua, crear materiales educativos, y un largo etcétera que supone levantar acta de la premisa de una motivación permanente por mejorar la práctica docente y la promesa de un futuro en el que la comunicación personal, en lenguas diversas pero igualmente entrañables para sus hablantes, atraviese fronteras y contribuya a crear ciudadanos más maduros y personas más humanas.

Y es que el doble, y doblemente marcado, componente relacional de las lenguas, y de cualquier proceso educativo que se defina como tal, satisface una tendencia intrínsecamente humana, comporta una gratificación difícilmente mensurable y es una fuente de estímulo y de honda satisfacción académica y profesional.

La sociedad actual necesita, cada vez más, ciudadanos competentes que sean capaces de desenvolverse en múltiples y variadas situaciones, contextos y realidades. Esto hace que la educación de niños y jóvenes deba ser planteada desde un enfoque educativo diferente en el que su único cometido no sea sólo aprender sino también desarrollar, adquirir y poner en práctica diferentes competencias, esto es, habilidades, destrezas, estrategias y actitudes. En definitiva, se busca que los alumnos puedan aplicar los conocimientos escolares en contextos reales y diferentes de aquellos en los que, inicialmente, fueron aprendidos (Iglesias, 2009, 9).

Esa nueva ciudadanía sólo podrá surgir de oportunidades educativas cuyo germen dependerá en buena medida del elenco de experiencias y encuentros culturales vivos y significativos que se proporcionen en el ámbito de la formación del profesorado, y, dentro de ella, en los programas de prácticas docentes. Ejemplos como los anteriores señalan algunas de las muchas posibilidades para que el Practicum pueda llegar a ser no sólo un paso previo a la cualificación profesional reglada, sino un espacio de encuentros en la palabra y en la acción, un lugar de reconocimiento y descubrimiento mutuo, un taller de experimentación educativa, de crecimiento cívico y de creación cultural y un dinamizador de proyectos de 
convivencia y aprendizaje cooperativo que, generados en una matriz de diversidad donde los otros se convierten en nuestros otros rostros, tengan plena relevancia y sean forjadores de verdadera "común unidad", esto es, de verdadera humanidad.

\section{REFERENCIAS BIBLIOGRÁFICAS}

Aguado Odina, M. ${ }^{a}$ T. (2010) Diversidad cultural y logros de los estudiantes en educación obligatoria: lo que sucede en las escuelas. Madrid, Subdirección General de Documentación y Publicaciones-Ministerio de Educación.

Austin, J. L. (1982) Cómo hacer cosas con palabras: Palabras y acciones. Barcelona, Paidós.

BhabHa, H. K. (1990) Nation and Narration. London and New York, Routledge.

Buitrago Gómez, M. ${ }^{a}$ C. (2009) La pluralidad lingüística: aportaciones sociales, culturales y formativas. Madrid, Subdirección General de Documentación y Publicaciones-Ministerio de Educación.

Byram, M.; Gribkova, B. y STARKey, H. (2002) Developing the Intercultural Dimension in Language Teaching. A practical introduction for teachers. Strasbourg: Language Policy Division Directorate of School, Out-of-School and Higher Education DGIV Council of Europe. Consultado el 12 de julio de 2011. http://www.coe.int/t/dg4/linguistic/source/ guide_dimintercult_en.pdf.

Casassus, J. (2004) Prefacio, en Naranjo, C. Cambiar la educación para cambiar el mundo. Vitoria-Gasteiz, La Llave D. H.

Claire, H. (2006) Education for Cultural Diversity and Social Justice, en ARTHUR, J.; GrAAINER, T. y WRAY, D. (eds.) Learning to Teach in the Primary School. London/New York, Routledge, 307-317.

CORTINA, A. (2007) Ética de la razón cordial. Educar en la ciudadanía en el siglo XXI. Oviedo, Nobel.

COUNCIL OF EuROPE (2001) Common European Framework of Reference for Languages: Learning, Teaching, Assessment. Strasbourg: Language Policy Division. Consultado el 12 de julio de 2011. http://www.coe.int/t/dg4/linguistic/cadre_en.asp.

Davies Samway, C. y MCKeOn, D. (eds.) (1993) Common Threads of Practice: Teaching English to Children Around the World. Alexandria, TESOL.

Dobson, A.; PÉrez, M. ${ }^{a}$ D. y Johnstone, R. (2010) Programa de Educación Bilingüe en España. Informe de la evaluación: Resultados de la evaluación independiente sobre el Proyecto de Educación Bilingüe del Ministerio de Educación y el British Council en España. Madrid, British Council-Ministerio de Educación.

DurÁn MarTínez, R. (2011) Spanish and British Teacher Trainees on Exchange in Primary Schools Abroad: an Intercultural Experience in Educational Settings. Porta Linguarum, 15, 115-133.

Durán, R.; GutiérRez, G. y Beltrán, F. (2006) Guía Internacional de Prácticas Docentes en Centros de Enseñanza Primaria/International Guide for Placement Abroad in Primary Schools. Salamanca, Universidad de Salamanca.

EURYDICE (2006) Aprendizaje Integrado de Contenidos y Lenguas (AICLE) en el contexto escolar europeo. Madrid, Secretaría General Técnica del Ministerio de Educación y Ciencia.

- (2009) Autonomía y responsabilidades del profesorado en Europa. Madrid, Secretaría General Técnica del Ministerio de Educación. 
- (2010) Key Data on Teaching Languages at School in Europe. Brussels, Educational, Audiovisual and Culture Executive Agency. Consultado el 12 de julio de 2011. http:// www.docstoc.com/docs/37622040/Eurydice-Update--Key-Data-on-Teaching-Languages-at-School-in-Europe

Fernández Batanero, J. M. ${ }^{a}$ (2009) Un currículum para la diversidad. Madrid, Síntesis.

García Carrasco, J. (2006) Los procesos formativos y el sistema emocional. Teoría de la Educación, 18, 27-53.

Goldsmith, M. (2006) Try feedforward instead of feedback, en GoldSMith, M. y LYONS, L. (eds.) Coaching for leadership. San Francisco, Pfiffer, 45-50.

GONZÁlez PÉREZ, I. (2011) Desarrollo y cultura tras los objetivos del milenio: consecuencias en la cooperación educativa internacional. Revista Española de Educación Comparada, 17, 31-64.

GonZÁlez, M.; Guillén, C. y Vez, J. M. (2010) Didáctica de las lenguas modernas: competencia plurilingüe e intercultural. Madrid, Síntesis.

GrinBlat, J. A. (2010) Introducción: La inmigración, un asunto de importante preocupación, en Blandin, C. (ed.) Atlas de las migraciones. Las rutas de la humanidad. Valencia, Le Monde Diplomatique, Akal, UNED-Fundación MonDiplo, 8-9.

GutiérRez, G.; BeLtrán, F. y DuRÁn, R. (2010) Un proyecto de aprendizaje integrado de contenidos y Lengua Inglesa: Cristóbal Colón/A content and language integrated learning Project: Christopher Columbus. Salamanca, Universidad de Salamanca.

Iglesias Rodríguez, A. (2009) Enseñar por competencias. Programación y evaluación en Educación Primaria y en Educación Secundaria Obligatoria. Salamanca, Degratis Editores, S.L.

Kelly, M.; Grenfell, M.; Allan, R.; Kriza, C. y McEvoy, W. (2004) European Profile for Language Teacher: A frame of reference. European Commission Directorate General for Education and Culture, University of Southampton.

Lasagabaster, D. y Ruiz de ZARobe, Y. (eds.) (2010) CLIL in Spain: Implementation, results and teacher training. Cambridge, Cambridge Scholars Publishing.

López-Seivane, F. (2007) Cosas que aprendi de Oriente. Madrid, Oberon.

MARTINELLI, S. y TAYLOR, M. (2010) Aprendizaje Intercultural. Madrid, Subdirección General de Documentación y Publicaciones, Ministerio de Educación.

MAYOR ZARAgOZA, F. (1999) Los nudos gordianos. Barcelona, Galaxia Gutenberg-Círculo de Lectores.

Merrill Valdés, J. (1986) Culture Bound. New York, Cambridge University Press.

MORROw, K. (2004) Insights from the Common European Framework. Oxford, Oxford University Press.

ODENIYI, V. y LAZAR, G. (2011) A cross-cultural awareness workshop: language, communication and difference. The Teacher Trainer, 25 (1), 2-8.

ORDEN ECI/3854/2007, de 27 de diciembre, por la que se establecen los requisitos para la verificación de los Títulos Universitarios oficiales que habiliten para el ejercicio de la profesión de Maestro en Educación Infantil. BOE, de 29 de diciembre de 2007, n. ${ }^{\circ}$ 312.

ORDEN ECI/3857/2007, de 27 de diciembre, por la que se establecen los requisitos para la verificación de los Títulos Universitarios oficiales que habiliten para el ejercicio de la profesión de Maestro en Educación Primaria. BOE, de 29 de diciembre de 2007, n. ${ }^{\circ} 312$.

Orland-Barak, L. (2011) Professional Learning in Practice: Orientations and Methods, en Raposo, M.; Martínez, M. E.; Muñoz, P. C.; Pérez, A. y Otero, J. C. (coords.) 
Evaluación y supervisión del practicum: el compromiso con la calidad de las prácticas. Santiago de Compostela, Andavira, 48.

PEDRAZ GÓmez, T. (2009) Las lenguas extranjeras como vehículo de comunicación intercultural. Madrid, Subdirección General de Documentación y Publicaciones-Ministerio de Educación.

RÁbano Llamas, M. F. (2010) La Competencia Intercultural. Una investigación en el Aula de Inglés de Primaria. Guadalajara, Aache Ediciones.

Ruiz de Zarobe, Y. y JimÉnez CATALÁN, R. M. (2009) Content and Language Inegrated Learning: Evidence from research in Europe. Clevedon, Multilingual Matters.

SEN, A. (2006) Identity and Violence. The Illusion of Destiny. London, Allen Lane.

Thomas, G. (2011) How to do your Case Study: A Guide for Students and Researchers. London, Sage.

Tomlinson, C. A. (2005) Estrategias para trabajar con la diversidad en el aula. Buenos Aires, Paidós.

UNESCO (2000) Foro Mundial sobre la Educación. Marco de Acción de Dakar. Educación para Todos: cumplir nuestros compromisos comunes. Francia.

Vez JeREMías, J. M. (2001) La Investigación en Didáctica de las Lenguas Extranjeras. Educatio Siglo XXI, 29 (1), 81-108.

Vez JeREmías, J. M. y GonZÁlez PiÑeiro, M. (2004) Intercultural competence and the European Dimension, en MAdrid, D. y McLaren, N. (eds.) TEFL in Primary Education. Granada, Ediciones Universidad de Granada, 341-383.

Vila Merino, E. S. (2005) Mundo de la vida y cultura: la educación como acción ética e intercultural. Teoría de la Educación, 17, 81-96.

WoOdGate-Jones, A. (2008) Training confident primary modern foreign language teachers in England: An investigation into preservice teachers' perceptions of their subject knowledge. Teaching and Teacher Education, 24 (1), 1-13.

Zabalza BerazA, M. (2011) Evaluar la calidad del Practicum: una propuesta, en Raposo, M.; Martínez, M. E.; Muñoz, P. C.; Pérez, A. y Otero, J. C. (coords.) Evaluación y supervisión del practicum: el compromiso con la calidad de las prácticas. Santiago de Compostela, Andavira, 101-128.

Zander Stone, R. y Zander, B. (2002) The Art of Possibility. Transforming Professional and Personal Life. New York, Penguin.

ZuBIRI, X. (1980) Inteligencia Sentiente: Inteligencia y razón. Madrid, Alianza Editorial. 